\title{
Caratterizzazione microstrutturale e prove di resilienza su giunti Friction Stir Welding e Linear Friction Welding di compositi a matrice metallica
}

\author{
L. Ceschini, A. Morri, F. Rotundo \\ SMETEC, Università degli Studi di Bologna; \\ ceschini@bomet.fci.unibo.it, alessandro.morri@bomet.fci.unibo.it,fabio.rotundo@unibo.it
}

G.L. Garagnani, M. Merlin

Dipartimento di Ingegneria, Università degli Studi di Ferrara

glgaragnani@ing.unife.it,mattia.merlin@unife.it

RIASSUNTO. In questo studio sono stati caratterizzati giunti Friction Stir Welding e Linear Friction Welding su compositi a matrice in lega di alluminio e rinforzo particellare ceramico. Il processo FSW è stato applicato a due compositi ottenuti con processo fusorio, quindi estrusi e trattati termicamente T6: AA6061/20\%vol. $\mathrm{Al}_{2} \mathrm{O}_{3 \mathrm{p}} \mathrm{e}$ AA7005/10\%vol. $\mathrm{Al}_{2} \mathrm{O}_{3 \mathrm{p}}$. I giunti $\mathrm{LFW}$ sono stati invece realizzati su un composito con matrice in lega di alluminio e rinforzo particellare in carburo di silicio, ottenuto mediante metallurgia delle polveri, quindi forgiato e trattato termicamente T4: AA2124/25\%vol.SiC . Sono stati esaminati gli effetti della saldatura sulle caratteristiche microstrutturali dei giunti, avvalendosi di tecniche di microscopia ottica con analisi di immagine e di microscopia elettronica in scansione (SEM) con microsonda a dispersione di energia (EDS). Sono state quindi condotte prove di resilienza con pendolo strumentato Charpy. Lo studio dei meccanismi di danneggiamento è stato effettuato mediante analisi al SEM delle superfici di frattura. Entrambi i processi di saldatura hanno portato a giunti sostanzialmente esenti da difetti. La microstruttura dei cordoni è risultata dipendente sia dalle caratteristiche microstrutturali iniziali dei compositi considerati, sia dalla tipologia di processo di saldatura. Nel caso dei compositi AA6061/20\% $\mathrm{Al}_{2} \mathrm{O}_{3 \mathrm{p}}$ e $\mathrm{AA} 7005 / 10 \% \mathrm{Al}_{2} \mathrm{O}_{3 \mathrm{p}}$ saldati FSW si è osservato un sostanziale incremento di resilienza, rispetto al materiale base, in conseguenza dell'affinamento dei grani della matrice, della riduzione della dimensione media delle particelle di rinforzo e della loro spigolosità, indotte dal processo di saldatura. Il composito AA2124/25\% SiC $\mathrm{p}_{\mathrm{p}}$ saldato LFW ha presentato valori di resilienza confrontabili con quelli del materiale base, in conseguenza, soprattutto, dei limitati effetti della saldatura su dimensione e distribuzione delle particelle di rinforzo.

AbStRact. This paper presents the effects of Friction Stir Welding (FSW) and Linear Friction Welding (LFW) on aluminium based metal matrix composites (MMCs) containing a ceramic particulate reinforcement. The FSW process has been carried out on two types of composite obtained by means of stir casting, extruded and T6 heat-treated: AA6061/20\%vol. $\mathrm{Al}_{2} \mathrm{O}_{3 \mathrm{p}}$ and AA7005/10\%vol. $\mathrm{Al}_{2} \mathrm{O}_{3 \mathrm{p}}$. LFW joints have been realized on an aluminum based MMC obtained by powder metallurgy, forged and T4 heat-treated: AA2124/25\%vol.SiC . Optical Microscopes (OM) and Scanning Electron Microscopes (SEM) with Energy Dispersive Spectroscopy (EDS) microprobe have been used to characterize the effects of the welding processes on the microstructural properties of the joints. Instrumented impact strength tests have been carried out on Charpy samples and fractographic examination has been performed by SEM analysis of fracture surfaces. Both the welding 
processes permitted to obtain joints substantially defect-free. The microstructures of the joints were found to be dependent on both the initial microstructure of the composites and the welding process. With respect to the base metal, an increase on impact strength properties of $\mathrm{AA} 6061 / 20 \% \mathrm{Al}_{2} \mathrm{O}_{3 \mathrm{p}}$ and $\mathrm{AA} 7005 / 10 \% \mathrm{Al}_{2} \mathrm{O}_{3 \mathrm{p}}$ joined by FSW have been measured, due to the reduction of the grain size, aspect ratio and mean size of the reinforcement particles. The impact strength of the base $\mathrm{AA} 2124 / 25 \% \mathrm{SiC}_{\mathrm{p}}$ composite and $\mathrm{LFW}$ joints were found to be comparable, due to the limited effects of the welding process on the microstructure, which was already very fine in the base material.

Parole Chiave. Compositi a matrice metallica; Alluminio; Saldatura; Friction Stir Welding; Linear Friction Welding; Microstruttura; Resilienza.

\section{INTRODUZIONE}

I compositi a matrice metallica (CMM) e rinforzo particellare ceramico possono essere saldati utilizzando le stesse tecnologie applicate alle corrispondenti leghe non rinforzate. L'ottenimento di giunzioni affidabili è tuttavia dipendente dai parametri di processo, che devono essere attentamente controllati, soprattutto nel caso di processi di saldatura per fusione, al fine di evitare la formazione di difetti microstrutturali nel cordone. In processi TIG e MIG, ad esempio, la presenza del rinforzo particellare può creare problematiche peculiari, quali: formazione di fasi infragilenti, a seguito di indesiderate reazioni interfacciali tra matrice e rinforzo; aumento della viscosità del bagno, con conseguente riduzione di penetrazione del cordone di saldatura; disomogenea distribuzione del rinforzo; presenza di gas occluso [1-2]. I limiti delle tecnologie di saldatura per fusione possono essere superati applicando processi di giunzione allo stato solido, quali la Friction Stir Welding (FSW) e la Linear Friction Welding (LFW), che permettono di eliminare tutte le problematiche connesse alla fusione della matrice [3-5]. Nella FSW (Fig. 1-a) un utensile cilindrico, composto da una spalla e da un pin, opportunamente sagomato, viene posizionato all'altezza della linea di giunzione dei componenti, che devono essere rigidamente accostati, quindi posto in rotazione e fatto traslare lungo la linea di giunzione. Al fine di controllare le caratteristiche microstrutturali dei giunti e, conseguentemente, di aumentare le loro proprietà meccaniche, è necessario ottimizzare i parametri di saldatura: forma e dimensioni del pin, velocità di rotazione e di traslazione, carico applicato all'utensile. L'attrito che si genera nel contatto tra l'utensile ed il materiale, nel suo moto di roto-traslazione lungo la linea di giunzione, provoca un riscaldamento localizzato senza che venga mai raggiunta la temperatura di fusione, favorendo alti tassi di deformazione e il rimescolamento plastico dei lembi di saldatura [6-8]. La FSW è stata inizialmente sviluppata per la saldatura di leghe di alluminio, ma studi recenti hanno dimostrato come essa possa essere applicata con successo anche ai relativi compositi con rinforzo particellare [9-10].

Uno dei possibili problemi nell'applicazione della FSW ai CMM con rinforzo discontinuo è tuttavia costituito dall'usura dell'utensile, indotta dalla presenza di particelle ceramiche di elevata durezza [11]. Questa problematica può essere superata grazie alla saldatura lineare per attrito LFW, di cui si riporta uno schema in Fig. 1-b. Questa possibile alternativa alla FSW è nata con l'obiettivo di estendere l'applicazione della saldatura per attrito rotante (Rotary Friction Welding, RFW) a componenti non assial-simmetrici e di forma complessa [12].
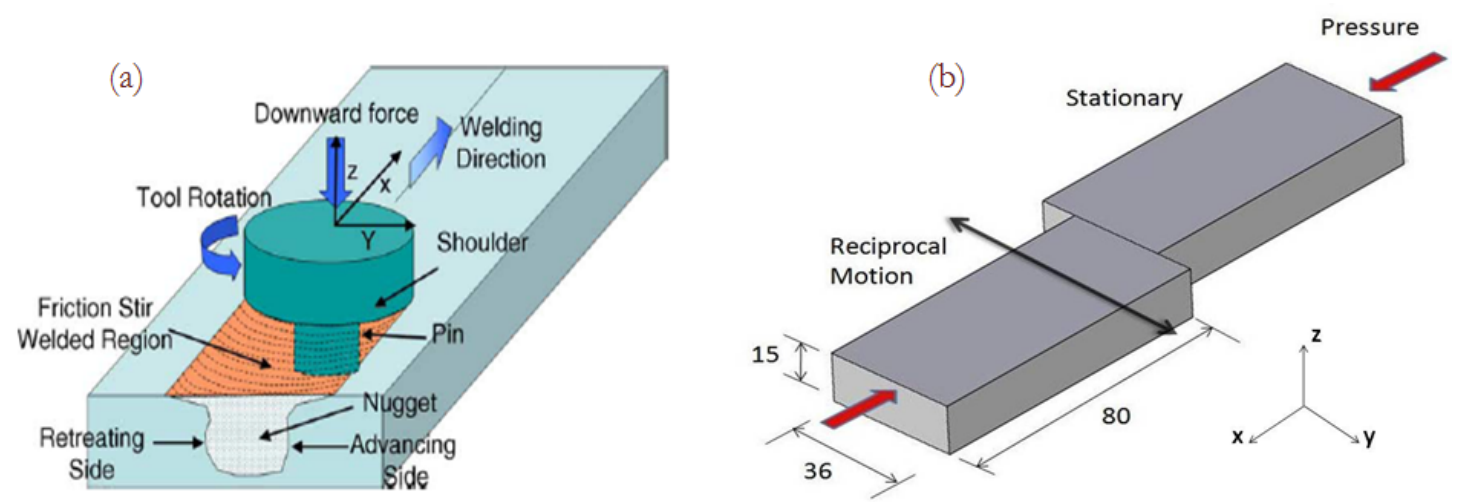

Figura 1: Rappresentazione schematica dei processi (a) FSW [7] e (b) LFW [11]. 
Nella LFW i due componenti sono posti a contatto, sottoposti ad una spinta assiale e quindi posti in moto lineare reciproco alterno. Il calore generato per attrito determina una diminuzione di resistenza al flusso plastico del materiale ed una conseguente deformazione localizzata delle superfici, che conduce ad un incremento dell'area reale di contatto. All'aumentare della spinta assiale, il materiale all'interfaccia si deforma, iniziando a fuoriuscire dai lembi della saldatura. $\mathrm{Si}$ ha quindi la formazione di un uniforme cordone di saldatura, costituito dal materiale plasticizzato, parte del quale viene espulso all'interfaccia (flash), e un conseguente accorciamento assiale dei componenti. Raggiunto un prefissato accorciamento assiale (burn-off), il moto viene rapidamente arrestato e la forza di contatto viene mantenuta per consolidare la saldatura, che dura in totale pochi secondi [12]. I principali parametri di processo per la LFW sono: frequenza e ampiezza di oscillazione, spinta ed accorciamento assiale. Le applicazioni delle LFW sono state sviluppate soprattutto in campo aeronautico, con applicazioni quali la giunzione di rame e alluminio per la realizzazione di conduttori, oltre alla saldatura rotore-paletta degli stadi a bassa pressione del compressore, realizzati in leghe di titanio. Ad oggi, la applicazione della LFW ai CMM è stata solo oggetto di studi preliminari $[5,13]$.

Obiettivo del presente lavoro è stato quello di valutare gli effetti dei processi di FSW e LFW sulla microstruttura di compositi, a matrice in lega di alluminio e rinforzo particellare ceramico, avvalendosi di tecniche di microscopia ottica con analisi di immagine, oltre che di microscopia elettronica in scansione. Sono stati esaminati, in particolare, giunti FSW sui compositi W6A20A (AA6061/20\%vol. $\mathrm{Al}_{2} \mathrm{O}_{3 \mathrm{p}}$ ) e W7A10A (AA7005/10\%vol. $\mathrm{Al}_{2} \mathrm{O}_{3 \mathrm{p}}$ ), e giunti LFW sul composito AMC225xe (AA2124/20\%vol.SiC ). Sui giunti e, per confronto, sui rispettivi materiali base, sono state inoltre condotte prove di resilienza strumentata. Lo studio delle superfici di frattura è stato effettuato mediante analisi al microscopio elettronico in scansione.

\section{MATERIALI E METODI}

I giunti FSW sono stati realizzati su piastre di materiale composito W6A20A (AA6061/20\%vol. $\mathrm{Al}_{2} \mathrm{O}_{3 \mathrm{p}}$ ) e W7A10A (AA7005/10\%vol. $\mathrm{Al}_{2} \mathrm{O}_{3 \mathrm{p}}$ ). Le composizioni chimiche nominali delle rispettive matrici sono riportate in Tab. I. Entrambi i compositi sono stati prodotti da Duralcan con processo fusorio di Compocasting [14], quindi estrusi a $480{ }^{\circ} \mathrm{C}$ fino all'ottenimento di piastre aventi sezione $100 \times 7 \mathrm{~mm}^{2}$, infine trattati termicamente T6. Le piastre sono state saldate mediante Friction Stir Welding presso il GKSS Research Institute (Geesthacht, Germania), utilizzando un utensile in Ferro-Titanit (trattato termicamente a $63 \mathrm{HRC}$ ), con spalla del diametro di $18 \mathrm{~mm}$ e pin con lavorazione elicoidale sinistrorsa (diametro $8 \mathrm{~mm}$ e lunghezza $6.8 \mathrm{~mm}$ ). La saldatura è stata realizzata applicando una forza verticale di $12 \mathrm{kN}$, una velocità di rotazione di $600 \mathrm{rpm}$ e una velocità di traslazione di $300 \mathrm{~mm} / \mathrm{min}$.

I giunti LFW sono stati realizzati sul composito AMC225xe, con matrice in lega d'alluminio 2124 (Tab. II), rinforzata con il $25 \%$ in volume di particelle fini di SiC $(\leq 3 \mu \mathrm{m})$. Il composito è stato prodotto da Aerospace Metal Composites Ltd (UK), mediante un processo di metallurgia delle polveri comprendente: miscelazione, evacuazione dei gas e successiva compattazione a caldo mediante pressatura isostatica. Le billette così prodotte sono state forgiate, con un rapporto di deformazione di 6:1, fino all'ottenimento di piatti con spessore di $15 \mathrm{~mm}$, successivamente trattati termicamente T4. Provini di sezione $15 \times 36 \mathrm{~mm}^{2}$ sono stati saldati mediante Linear Friction Welding presso The Welding Institute (TWI, Cambridge, UK). In questo caso i parametri utilizzati sono: spinta assiale $100 \mathrm{kN}$ (pressione risultante di $185 \mathrm{MPa}$ ), frequenza di oscillazione $50 \mathrm{~Hz}$, ampiezza di oscillazione $4 \mathrm{~mm}$, burn-off $2 \mathrm{~mm}$.

Sui giunti FSW e LFW non è stato eseguito alcun trattamento termico post-saldatura.

\begin{tabular}{ccccccccccc}
\hline & $\mathrm{Si}$ & $\mathrm{Fe}$ & $\mathrm{Cu}$ & $\mathrm{Mn}$ & $\mathrm{Mg}$ & $\mathrm{Zn}$ & $\mathrm{Ti}$ & $\mathrm{Cr}$ & $\mathrm{Zr}$ & $\mathrm{Al}$ \\
$\mathrm{AA} 6061$ & 0.65 & 0.15 & 0.18 & 0.10 & 0.97 & 0.009 & 0.02 & 0.19 & - & resto \\
$\mathrm{AA7005}$ & 0.25 & 0.24 & 0.08 & 0.43 & 1.33 & 4.59 & 0.03 & 0.13 & 0.13 & resto \\
\hline
\end{tabular}

Tabella I: Composizione chimica nominale (\% in peso) delle matrici in lega AA6061 e AA7005 dei compositi W6A20A (AA6061/20\%vol. $\mathrm{Al}_{2} \mathrm{O}_{3 \mathrm{p}}$ ) e W7A10A (AA7005/10\%vol. $\mathrm{Al}_{2} \mathrm{O}_{3 \mathrm{p}}$ ).

\begin{tabular}{cccccccccc}
\hline & $\mathrm{Si}$ & $\mathrm{Fe}$ & $\mathrm{Cu}$ & $\mathrm{Mn}$ & $\mathrm{Mg}$ & $\mathrm{Ti}$ & $\mathrm{Cr}$ & $\mathrm{Zn}$ & $\mathrm{Al}$ \\
$A A 2124$ & $\leq 0.20$ & $\leq 0.300$ & $3.80-4.90$ & $0.30-0.90$ & $1.20-1.80$ & $\leq 0.15$ & $\leq 0.100$ & $\leq 0.25$ & resto \\
\hline
\end{tabular}

Tabella II: Composizione chimica nominale (\% in peso) della matrice in lega AA2124 del composito AMC225xe.

La caratterizzazione microstrutturale dei giunti e dei materiali base è stata effettuata con tecniche di microscopia ottica $(\mathrm{OM})$, microscopia elettronica in scansione (SEM) con microanalisi a dispersione di energia (EDS) ed analisi di immagine 
(con software Image Pro-Plus). I campioni metallografici sono stati preparati con metodologie standard secondo ASTM E3 e quindi attaccati chimicamente con reagente Keller.

Le prove di resilienza sono state effettuate con un pendolo CEAST da $50 \mathrm{~J}$ dotato di mazza strumentata, idoneo per prove ad impatto su leghe leggere. Le proprietà di resilienza sono state misurate secondo normativa UNI EN ISO 14556:2003.

Nel caso dei giunti FSW sono stati realizzati provini Charpy V sub-size $\left(10 \times 5 \times 55 \mathrm{~mm}^{3}\right)$, aventi un intaglio con una profondità di $2 \mathrm{~mm}$ ed un raggio di raccordo al vertice di $0.25 \mathrm{~mm}$ ricavato nel centro della saldatura e parallelamente alla linea di avanzamento dell'utensile, secondo normativa ASTM E23. Sono stati realizzati 5 provini da ognuna delle saldature e altrettanti dai rispettivi materiali base.

Nel caso dei giunti LFW sono stati ricavati provini Charpy standard $\left(10 \mathrm{x} 10 \mathrm{x} 55 \mathrm{~mm}^{3}\right)$ con intaglio a $\mathrm{V}$ di profondità pari a $2 \mathrm{~mm}$ e raggio di raccordo $0.25 \mathrm{~mm}$, ricavato nel centro della saldatura e parallelamente alla linea di saldatura, secondo normativa ASTM E23. Sono stati realizzati 4 provini da ognuna delle saldature e altrettanti dai rispettivi materiali base.

\section{RISULTATI E DISCUSSIONE}

\section{Caratterizzazione microstrutturale}

T n Fig. 2 è riportata una immagine macro dei giunti FSW, che evidenzia, sulla superficie a contatto con la spalla dell'utensile, una finitura sostanzialmente analoga a quella indotta da una lavorazione di fresatura. La rugosità su tale lato è risultata pari a $\mathrm{R}_{\mathrm{a}}=3.5 \mu \mathrm{m}$, mentre sul lato opposto non sono state rilevate evidenti modificazioni di rugosità rispetto al materiale base $\left(\mathrm{R}_{\mathrm{a}}=0.7 \mu \mathrm{m}\right)$.
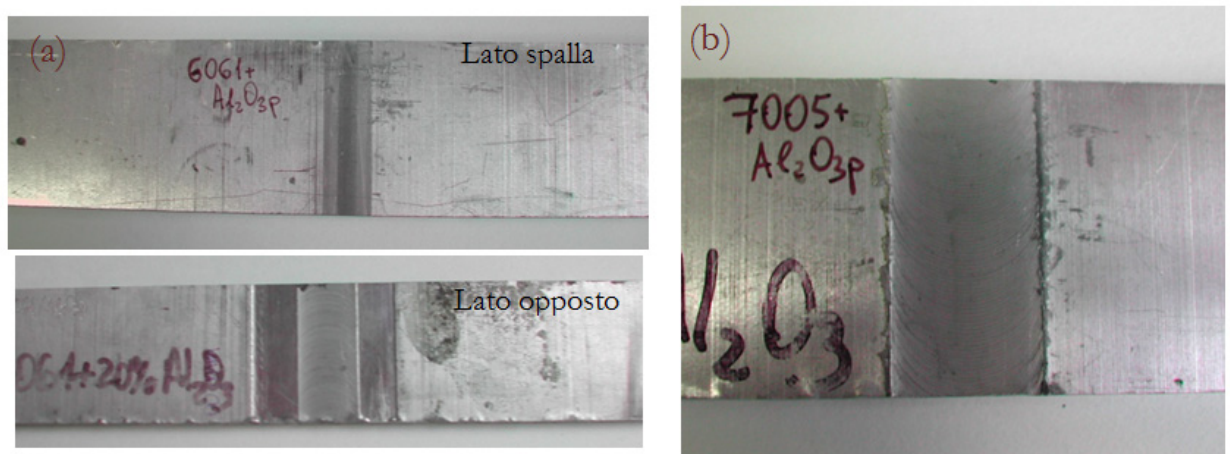

Figura 2: Morfologia dei giunti Friction Stir Welding su (a) W6A20A e (b) W7A10A.

Le immagini in microscopia ottica a basso ingrandimento della sezione trasversale dei cordoni FSW (Fig. 3) mostrano che la zona saldata può essere identificata solo per la presenza dei tipici onion rings, caratteristici della FSW, oltre che per una diversa distribuzione delle particelle di rinforzo, rispetto al materiale base.

(a)

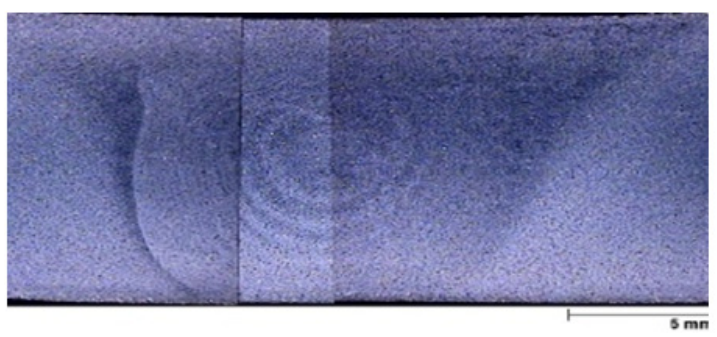

(b)

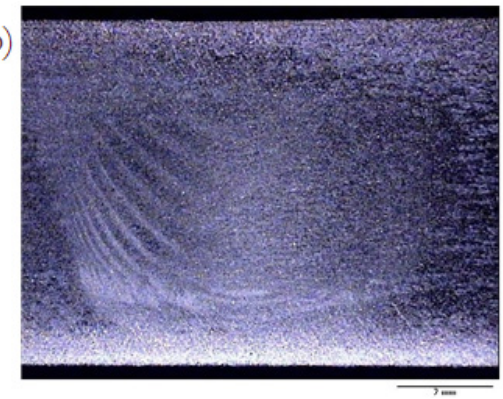

Figura 3: Micrografie ottiche delle sezioni trasversali dei giunti FSW sui compositi (a) W6A20A e (b) W7A10A.

È evidente la sostanziale assenza dei difetti tipici delle saldature per fusione dei CMM, quali porosità da gas o segregazione del rinforzo. Nel caso del composito W7A10A i giunti FSW hanno mostrato l'incompleta penetrazione della saldatura al centro della linea di giunzione, nella parte opposta a quella in contatto con la spalla dell'utensile (Fig. 4). 


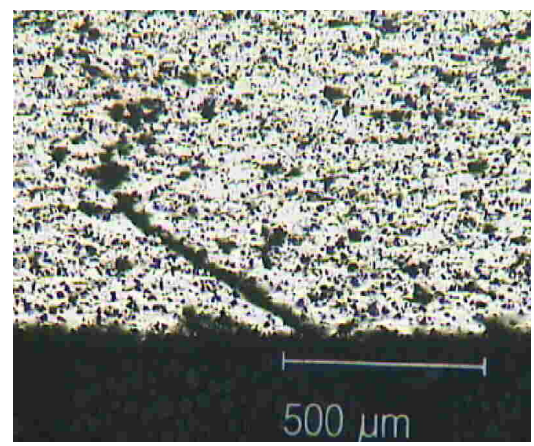

Figura 4: Difetto dovuto alla non completa penetrazione di saldatura sul lato opposto alla spalla dell'utensile nel giunto FSW sul composito W7A10A.

Micrografie ottiche a maggior ingrandimento della zona saldata FSW (Fig. 5) mostrano come, per effetto dell'azione di rimescolamento e abrasione esercitata dall'utensile di elevata durezza, si abbia una variazione sia nella distribuzione sia nella dimensione delle particelle di rinforzo.
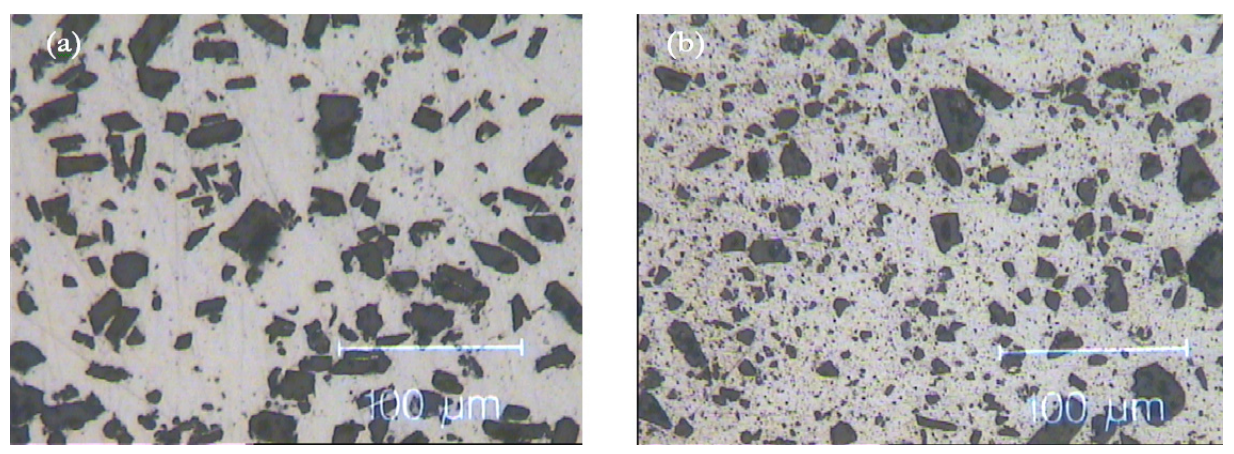

Figura 5: Micrografie ottiche del composito W6A20A: (a) materiale base; (b) centro del cordone FSW.

Questa osservazione è confermata dalle analisi di immagine, effettuate sulle micrografie ottiche; gli istogrammi di Fig. 6 mostrano una sostanziale riduzione dell'area media delle particelle di rinforzo, di circa il $50 \%$ nel caso del composito W6A20A e pari a circa il $30 \%$ per il W7A10A. La differenza tra i due compositi è da porsi in relazione alle diverse dimensioni iniziali delle particelle, che sono risultate essere mediamente di $135 \mu \mathrm{m}^{2}$ e $44 \mu \mathrm{m}^{2}$, rispettivamente. Per effetto dell'azione abrasiva esercitata dall'utensile, si è anche rilevata una riduzione della spigolosità delle particelle, con una conseguente riduzione dell'effetto di intensificazione degli sforzi, benefica dal punto di vista della tenacità. In conseguenza del rimescolamento plastico della matrice si è anche osservata una più omogenea distribuzione del rinforzo nella zona centrale del cordone (nugget), rispetto al materiale base.

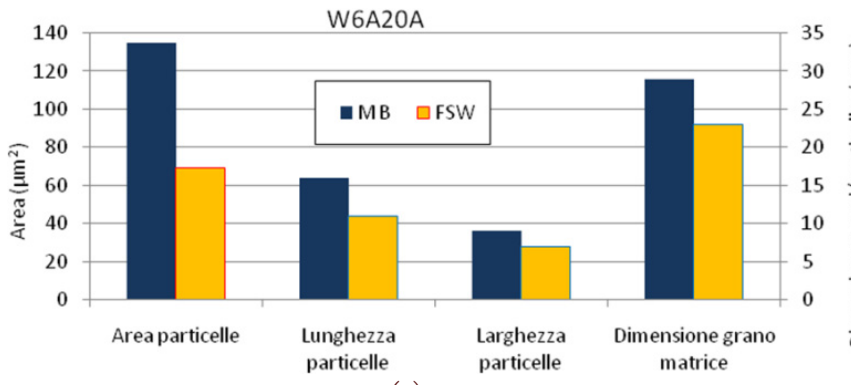

(a)

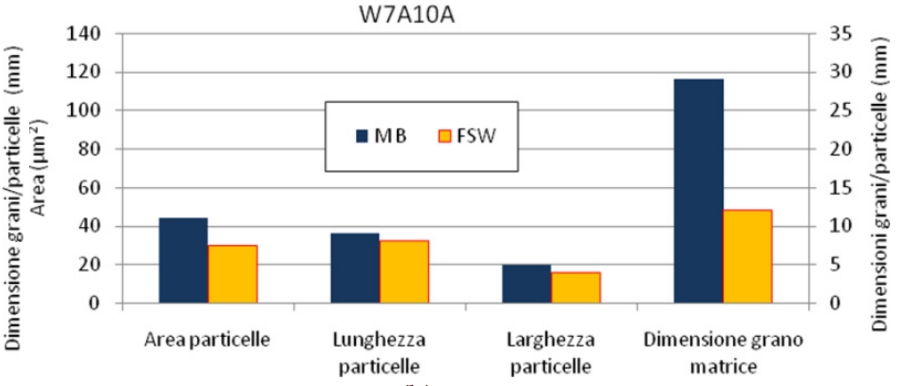

(b)

Figura 6: Confronto sulla dimensione delle particelle e sui grani della matrice di alluminio tra materiale base (MB) e giunti FSW per i compositi (a) W6A20A e (b) W7A10A.

Si è anche osservato un sostanziale affinamento dei grani della matrice nel nugget di entrambi i compositi, come illustrato negli istogrammi di Fig. 6 e nelle micrografie ottiche di Fig. 7, a causa dell'azione concomitante della severa deformazione 
plastica e del calore sviluppato per attrito, caratteristici della FSW. L'effetto di affinamento è da imputarsi alla ricristallizzazione dinamica della matrice indotta dal processo e favorita dalla presenza delle particelle di rinforzo, che agiscono da siti preferenziali di nucleazione. Nel composito W6A20A la dimensione media dei grani è diminuita da $29 \mu \mathrm{m}$ nel materiale base a $20 \mu \mathrm{m}$ nel nugget, mentre nel W7A10A da $29 \mu \mathrm{m}$ a $12 \mu \mathrm{m}$, rispettivamente. Il maggior affinamento dei grani rilevato per quest'ultimo composito può essere correlato ai maggior tassi di deformazione indotti dalla FSW, a causa della minore percentuale volumetrica di rinforzo nel materiale base.
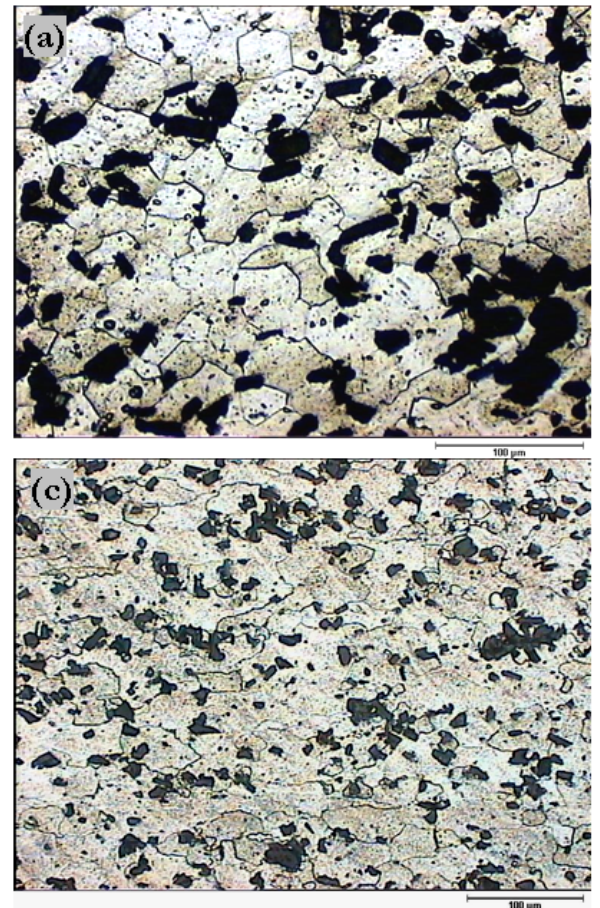
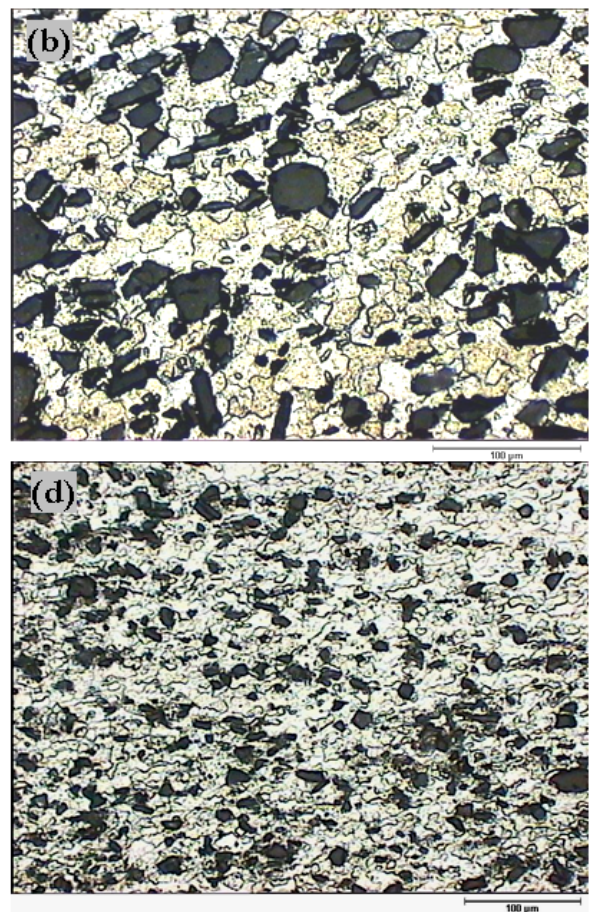

Figura 7: Effetto della FSW sulla dimensione dei grani della matrice di alluminio, per W6A20A:

(a) materiale base e (b) nugget, per W7A10A: (c) materiale base e (d) nugget.

La Fig. 8 mostra, invece, la morfologia dei giunti ottenuti dal processo LFW ed evidenzia la presenza del flash di materiale espulso durante la saldatura.

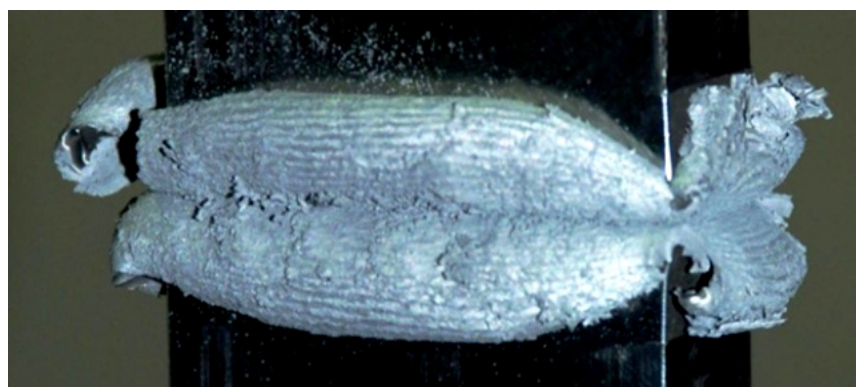

Figura 8: Aspetto del giunto ottenuto per LFW su AMC225xe.

L'analisi metallografica della sezione trasversale del giunto LFW, in microscopia ottica e luce polarizzata, ha messo in evidenza il rilevante flusso plastico a cui è sottoposto il materiale durante il processo e la conseguente fibrosità indotta nella matrice, come evidente dalla micrografia della sezione $x z$ (rispetto allo schema di Fig. 1) riportata in Fig. 9. Si distinguono una zona centrale (weld center), una zona termo-meccanicamente alterata (TMAZ) ed una zona termicamente alterata $(H A Z)$.

Le ridotte dimensioni dei grani del materiale base non ne hanno permesso la risoluzione con le tecniche metallografiche disponibili. Tuttavia, confrontando la distribuzione delle particelle di rinforzo e l'orientazione delle bande prive di particelle, è possibile distinguere la deformazione plastica indotta nel materiale dal processo (Fig. 10). 


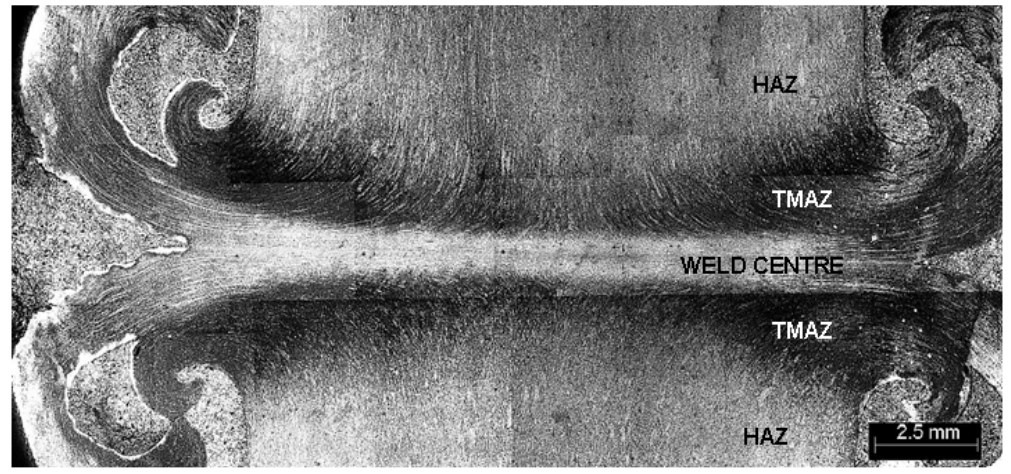

Figura 9: Micrografia a basso ingrandimento del cordone di saldatura LFW per AMC225xe.
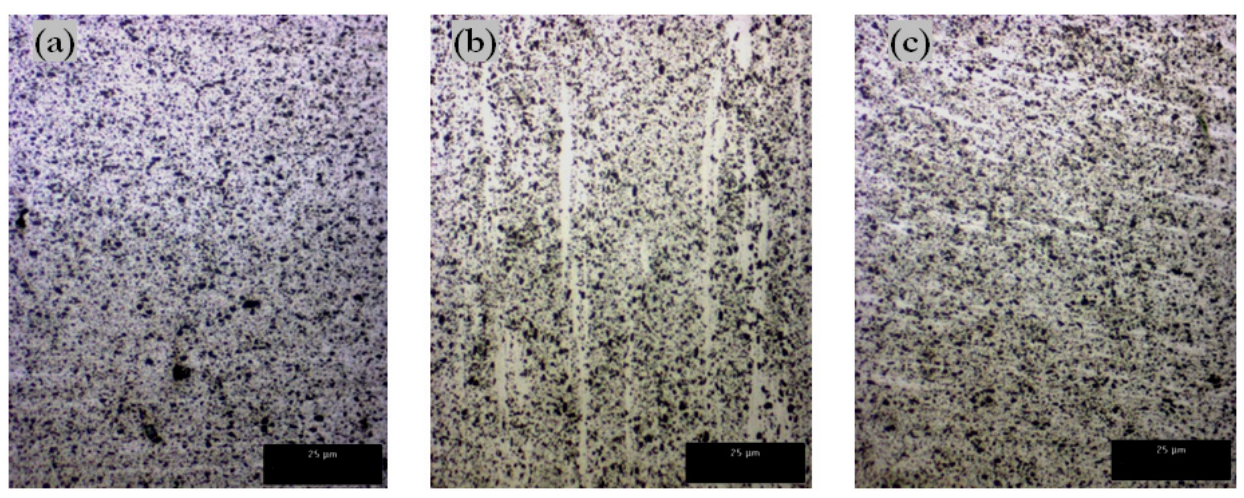

Figura 10: Distribuzione del rinforzo particellare nel composito AMC225xe saldato mediante LFW: (a) centro del cordone di saldatura, (b) materiale base, (c) TMAZ.

Se la zona centrale si caratterizza per una omogenea distribuzione delle particelle (Fig. 10-a), nella HAZ sono chiaramente distinguibili queste bande, dovute al processo di forgiatura cui è stato sottoposto il materiale base nel processo produttivo (Fig. 10-b), mentre nella TMAZ si può notare una struttura fortemente deformata nella direzione di espulsione del flash di saldatura (Fig. 10-c).

Dall'analisi di immagine delle micrografie SEM (i cui risultati sono illustrati nelle Fig. 11-12) è stato possibile osservare come la LFW non abbia prodotto effetti apprezzabili né sulla morfologia delle particelle di rinforzo (fattore di forma compreso fra 1.91 per il CMM base e 1.97 per la zona saldata), né sulle loro dimensioni, diversamente da quanto osservato per $\mathrm{i}$ giunti FSW. Nel caso dei giunti LFW, infatti, la mancanza dell'azione abrasiva dell'utensile, unita alle minori dimensioni iniziali delle particelle di rinforzo nel composito AMC225xe, ha infatti impedito o fortemente limitato la frattura delle particelle, nonostante la matrice venisse sottoposta a severa deformazione plastica. Occorre segnalare come la presenza di particelle di dimensioni molto ridotte nella distribuzione sia anche da attribuirsi alla preparativa metallografica, che comporta l'affioramento in superficie di porzioni di particelle di rinforzo sia per il materiale base sia per la zona di saldatura.
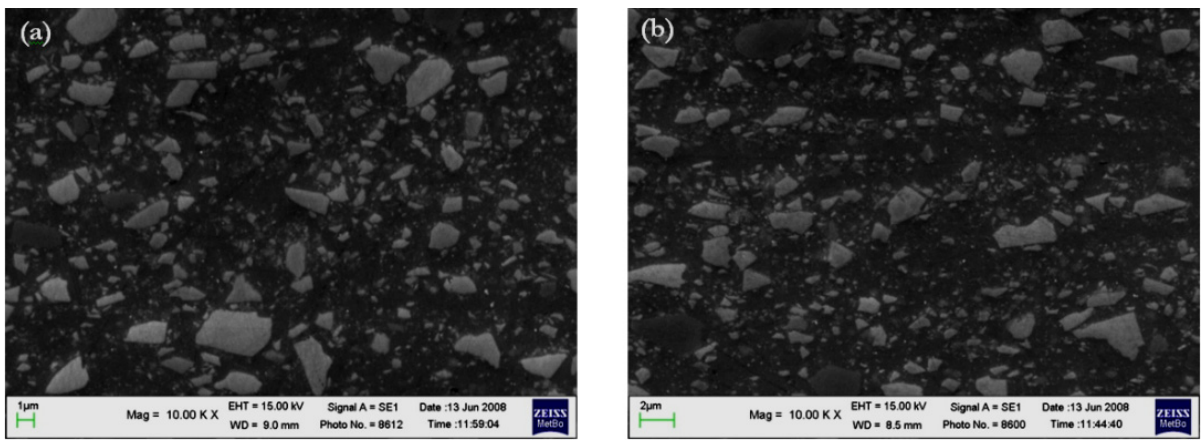

Figura 11: Micrografie SEM del composito AMC225xe saldato mediante LFW:

(a) materiale base, (b) centro della saldatura LFW. 


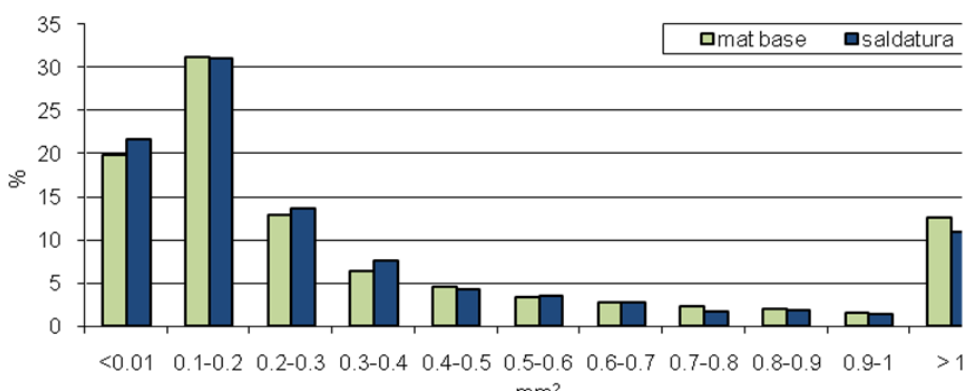

$\mathrm{mm}^{2}$

Figura 12: Distribuzione della dimensione delle particelle di rinforzo nel materiale base e nel cordone di saldatura LFW per il composito AMC225xe.

\section{Prove di resilienza}

I risultati delle prove di resilienza effettuate sui compositi saldati FSW e LFW, e sui corrispondenti materiali base, sono riportati in Fig. 13. In entrambi i compositi saldati FSW è interessante evidenziare come l'energia a frattura aumenti, rispetto al materiale base, da 0.7 a $2.7 \mathrm{~J}$ per W6A20A, e da $1.2 \mathrm{~J}$ a $2.9 \mathrm{~J}$ per W7A10A. Questo effetto può essere correlato alle variazioni microstrutturali indotte dalla FSW, precedentemente illustrate, ed in particolare all'azione simultanea dei seguenti fattori: affinamento e arrotondamento delle particelle; distribuzione più omogenea del rinforzo; riduzione della dimensione media dei grani della matrice. E' noto infatti l'effetto negativo, sulla resilienza dei compositi, di particelle di grandi dimensioni [10] e ad alta spigolosità, che agiscono da concentratori di tensione $[15,16]$. Anche la disomogenea distribuzione del rinforzo e la presenza di cluster di particelle hanno effetti negativi sulla resilienza ed è inoltre ben noto anche l'effetto positivo di un affinamento dei grani della matrice [10]. Dal confronto dei dati di resilienza (Fig. 13) con quelli relativi alle modificazioni microstrutturali indotte dalla FSW sui due compositi della sperimentazione (Fig. 6), si può dedurre come l'effetto migliorativo in termini di resilienza sia da attribuire in misura maggiore all'arrotondamento e alla diminuzione della dimensione delle particelle, che non all'affinamento del grano. Nel caso del composito W6A20A, ad esempio, in cui l'effetto della FSW sui grani della matrice è risultato di minore importanza rispetto a quello indotto sulle particelle di rinforzo, si è osservato un incremento più che rilevante di resilienza nella zona saldata dei giunti FSW rispetto al materiale base.

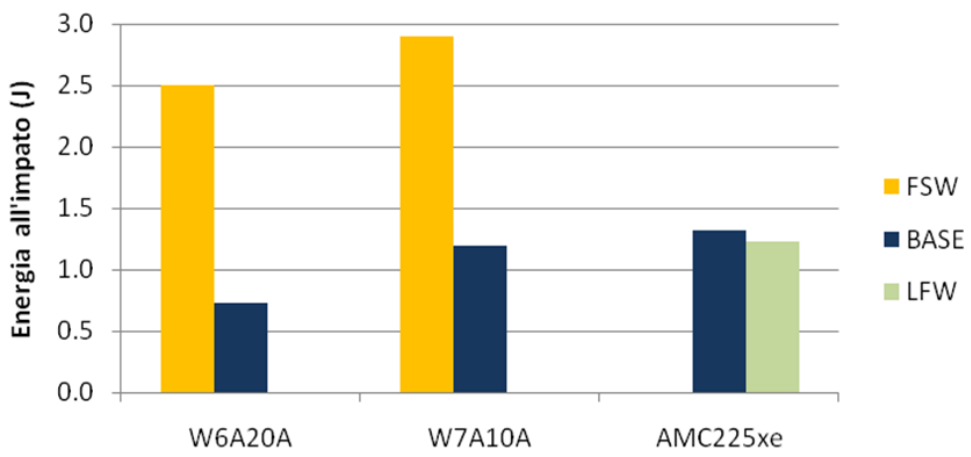

Figura 13: Risultati delle prove di resilienza relativi al materiale base e ai giunti FSW per $\mathrm{i}$ compositi W6A20A e W7A10A, e ai giunti LFW per il composito AMC225xe.

I risultati delle prove di resilienza sui giunti LFW (Figura 13) sul composito AMC225xe evidenziano, invece, un trascurabile effetto del processo di saldatura sulla resilienza: i campioni saldati hanno fornito un'energia totale ad impatto di $1.23 \mathrm{~J}$, mentre il materiale base di $1.32 \mathrm{~J}$, corrispondente ad una riduzione di solo il $7 \%$.

I diversi effetti indotti dalla FSW e LFW sulla resilienza dei compositi della sperimentazione sono da attribuire sia all'intrinseca diversità dei due processi di saldatura, sia alla diversa natura dei compositi utilizzati per realizzare i due tipi di giunzione. È bene infatti ricordare che il composito AMC225xe, utilizzato per i giunti LFW, è stato ottenuto per metallurgia delle polveri e risulta caratterizzato da una struttura di partenza molto fine, sia in termini di grani della matrice sia di dimensione delle particelle di rinforzo. Per contro, i CMM utilizzati per realizzare i giunti FSW sono stati prodotti con processo fusorio e risultano caratterizzati da grani e particelle di maggiori dimensioni. Nei giunti FSW, inoltre, la presenza di un utensile di elevata durezza ha indotto una elevata comminuzione delle particelle di rinforzo non presente nei giunti LFW. 
Superfici di frattura

L'esame frattografico delle superfici dei provini di resilienza ricavati dai giunti FSW ha evidenziato una maggiore deformazione plastica della matrice, rispetto ai provini ricavati dal materiale base (Fig. 14-15), mentre le superfici dei provini di resilienza ottenuti dai giunti LFW (Fig. 16) hanno mostrato una morfologia di frattura del tutto analoga a quella dei provini del materiale base.
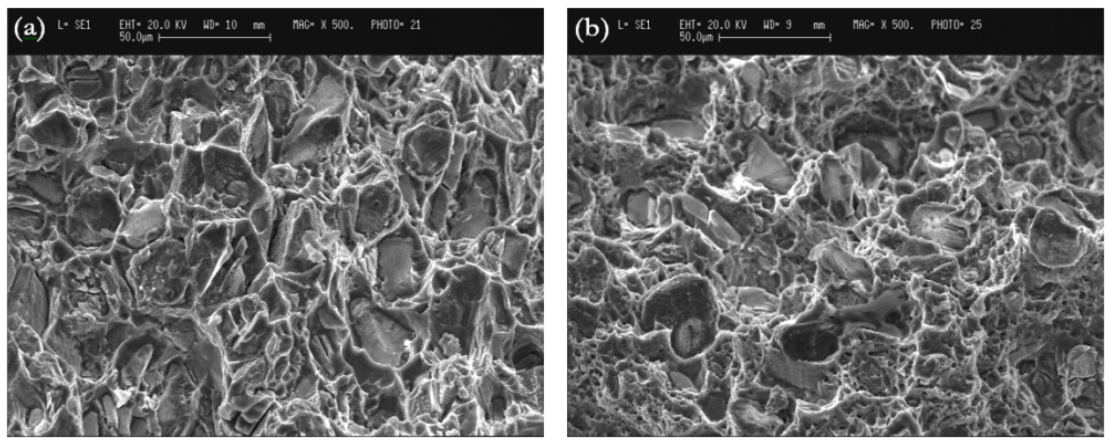

Figura 14: Micrografie SEM delle superfici di frattura dei provini di resilienza del composito W6A20A: (a) materiale base e (b) giunto FSW.
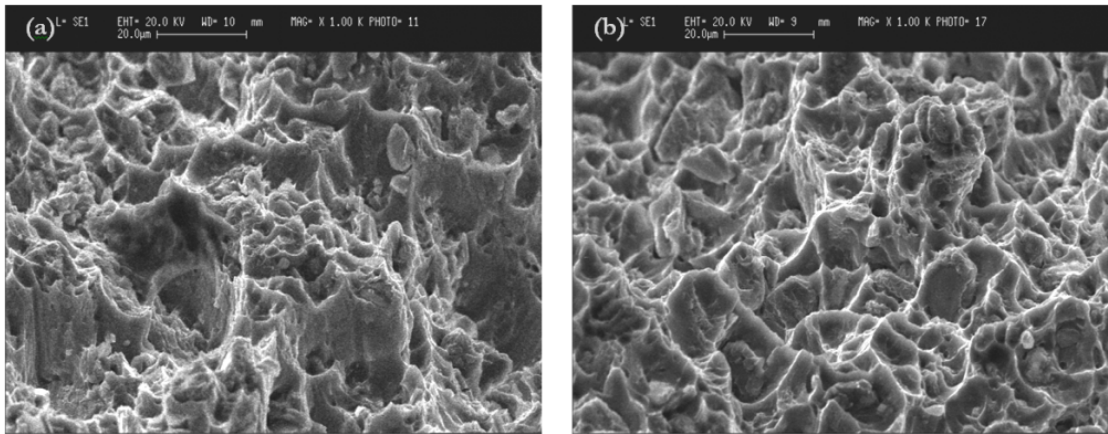

Figura 15: Micrografie SEM delle superfici di frattura dei provini di resilienza del composito W7A10A: (a) materiale base e (b) giunto FSW.
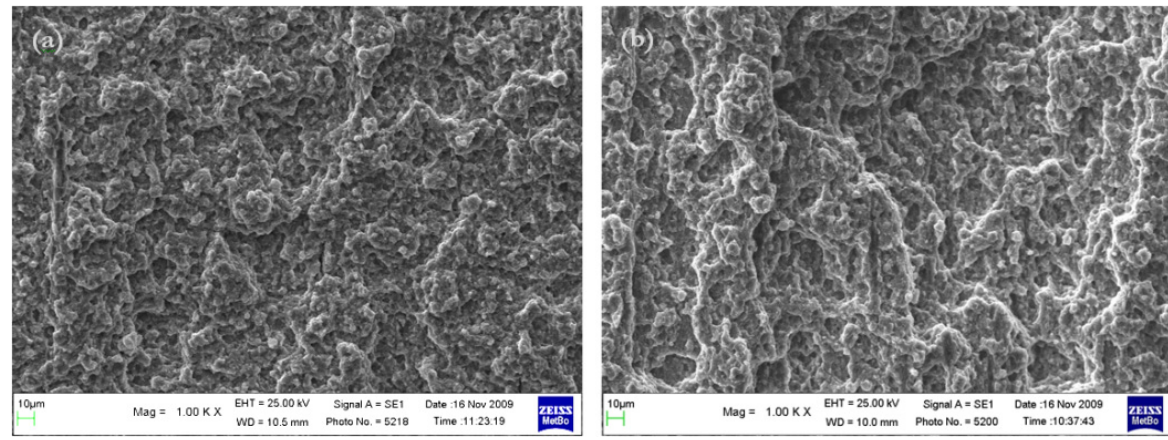

Figura 16: Micrografie SEM delle superfici di frattura dei provini di resilienza del composito AMC225xe: (a) materiale base e (b) giunto LFW.

I meccanismi di frattura evidenziati dalle analisi al SEM sono quelli tipici di compositi a matrice metallica e rinforzo particellare ceramico. E' possibile individuare zone con cedimento duttile della lega di alluminio, caratterizzate dallo sviluppo esteso di dimples e tear ridges, accanto a particelle fratturate (se di dimensioni rilevanti) o distaccate dalla matrice. La dimensione dei dimples e l'entità dei fenomeni di frattura o decoesione del rinforzo è risultata diversa per i vari compositi, in funzione della microstruttura iniziale del materiale base e di quella indotta dai processi di saldatura. La morfologia riscontrata nei provini saldati è in accordo con i risultati delle analisi microstrutturali e delle prove di resilienza, per entrambe le tipologie di giunti FSW e LFW. Nel caso dei giunti FSW, essa conferma gli effetti di affinamento indotti dal 
processo, sia sui grani della matrice sia sulle particelle di rinforzo, soprattutto per il composito W6A20A. Per contro, nel composito AMC225xe, l'effetto della saldatura LFW appare meno evidente.

In entrambi i giunti FSW e LFW, è possibile evidenziare un'ottima adesione tra matrice e rinforzo. Questo conferma l'assenza di reazioni interfacciali indesiderate che portano alla formazione di fasi infragilenti, caratteristiche dei processi di saldatura per fusione.

\section{CONCLUSIONI}

$\mathrm{N}$

el presente lavoro sono stati caratterizzati giunti Friction Stir Welding e Linear Friction Welding realizzati su compositi a matrice in lega di alluminio e rinforzo particellare. I giunti FSW sono stati prodotti su due compositi ottenuti con processo fusorio e trattati termicamente T6 (W6A20A e W7A10A); la LFW è stata invece applicata al composito AMC225xe, prodotto mediante metallurgia delle polveri e trattato termicamente T4.

- Entrambi i processi si sono dimostrati efficaci nel produrre giunti sostanzialmente esenti dai tipici difetti delle saldature convenzionali per fusione.

- La caratterizzazione microstrutturale dei giunti FSW ha evidenziato un notevole affinamento microstrutturale, in termini di riduzione della dimensione media dei grani, arrotondamento e riduzione della dimensione delle particelle di rinforzo. Si è inoltre riscontrata una più omogenea distribuzione del rinforzo nel cordone di saldatura.

- La LFW ha indotto limitati effetti sulla dimensione media delle particelle di rinforzo, sia per le ridotte dimensioni delle stesse nel materiale base, sia per l'assenza dell'utensile. Per quanto riguarda i grani della matrice, le tecniche di microscopia utilizzate non hanno consentito di apprezzare gli effetti del processo, anche se è lecito ipotizzare un elevato grado di affinamento dei grani nella zona centrale della saldatura.

- Le prove di resilienza hanno mostrato, nel caso dei giunti FSW, un miglioramento in termini di energia totale assorbita, rispetto al materiale base, correlabile alle modifiche microstrutturali precedentemente descritte. I giunti LFW hanno fornito valori di resilienza sostanzialmente confrontabili con quelli del materiale base, a conferma delle limitate modificazioni microstrutturali indotte dal processo.

- Le superfici di frattura hanno confermato le caratterizzazioni microstrutturali precedentemente descritte, mostrando inoltre un'ottima adesione matrice-rinforzo nei compositi saldati FSW e LFW in virtù dell'assenza di frasi infragilenti, tipiche di processi di saldatura per fusione.

\section{BIBLIOGRAFIA}

[1] T. W. Clyne, P. J. Withers, An Introduction to Metal Matrix Composites. Cambridge University Press, (1993).

[2] M. B. D. Ellis, International Materials Reviews, 2 (1996) 41.

[3] J. A. Wert, Scripta Materialia, 49 (2003) 607.

[4] L. Ceschini, I. Boromei, G. Minak, A. Morri, F. Tarterini, Composites Part A, 38 (2007) 1200.

[5] L. Ceschini, A. Morri, F. Rotundo, T.S. Jun, A. M. Korsunsky, Advanced Materials Research, 89-91 (2010) 461.

[6] C. J. Dawes, W. M. Thomas, Welding Journal, 75 (3) (1996) 41.

[7] R. S. Mishra, Z. Y. Ma, Materials Science and Engineering R, 50 (2005) 1.

[8] C. J. Dawes, Weld. and Metal Fabrication Journal, (1995).

[9] G. Minak, L. Ceschini, I. Boromei, M. Ponte, International Journal of Fatigue, 32 (1) (2010) 218.

[10] F. Bonollo, L. Ceschini, G. L. Garagnani, Applied Composite Materials, Kluwer Acad. Publ. NL, 4 (1997) 173.

[11] G. J. Fernandez, L. E. Murr, Materials Characterization, 52 (2004) 65.

[12] A. Vairis, M. Frost, Materials Science and Engineering A271 (1999) 477.

[13] T. S. Jun, F. Rotundo, L. Ceschini, A.M. Korsunsky, Key Engineering Materials, 385-387 (2008) 517.

[14] M. D. Huda, M. S. J. Hasmi, M. A. El-Baradie, Key Engineering Materials, 104-107 (1995) 37.

[15] S. G. Song, N. Shi, G. T. Gray III, J.A. Roberts, Metall. Mater. Trans., A27 (1996) 3739.

[16] P. Poza, J. Llorca, Metall. Mat. Trans. A, 26A (1995) 3131. 